\title{
PARA UNA ONTOLOGÍA DE LA HISTORIA: EL SUJETO DE LA HISTORICIDAD
}

«El tiempo, ese invento infernal que siempre nos arrastra» (Goethe)

\author{
José L. ARCE CARRASCOSO \\ Universidad de Barcelona
}

Resumen: El presente trabajo tiene como finalidad principal reflexionar acerca de la pregunta sobre el sentido ontologico de la historicidad y el tiempo de experiencia filosbfica que le corresponde. Para ello se lleva a cabo una contraposición entre las posturas de Dilthey y Heidegger, como respuestas extremas a una misma cuestion. Frente al relativismo psicológico y naturalizador del primero y, aun, frente al trascendentalismo idealista tradicional, el planteamiento de Heidegger surge como el polo opuesto, a pesar de que se puedan detectar en el algunas vacilaciones. Por ello, se impone volver a un punto intermedio que aune ambas posturas, proponiéndose una nueva lectura de la Estética trascendental de Kant, para considerar el tiempo como desconocida raíz común de carácter sintético a priori, en donde tienen su origen las dos caras, subjetiva y ontológica, de la temporalidad.

Abstract: Towads an ontology of history: the subject of historicity. The main objective of this paper is to reflect on the question of the ontological sense of historicity, and his corresponding time of philosophical experience. In so doing we contrast the positions adopted by Dilthey and Heidegger-starkly contrasting answers to the same question. Compared with the psicological and naturalising relativism of the former and, even compared with traditional idealist trascendentalism, Heidegger's approach is at the opposite extreme, despite a number of vacillations that have been detected in his argument. For this reason, we seek a return to the midle ground where both positions can be united, by proposing a new interpretation of Kant's trascendental aesthetics, in order to consider time as an unknown common root with a synthetic character a priori, in which lies the origin of the two faces of temporality, the subjective and the ontological. 


\section{Introducción}

Dado el carácter de la temática que intentamos abordar, quizas el primer requisito deba ser el de ponernos de acuerdo en un conjunto de presupuestos que de forma resumida podríamos recoger del siguiente modo. En primer lugar, ya que hablamos del sujeto de la historicidad, conviene recordar que la metafísica del sujeto ha venido desenvolviendo, a la vez que transformándose desde un estadio conciencialista a otro momento en el que la subjetividad para ser entendida de forma adecuada debe quedar definida dentro de unos márgenes intersubjetivos que posibiliten su propia existencia. Y por cuanto al tema de la historia de la metafísica, de forma paralela, también cabría reconocer que el mismo sujeto del devenir histórico es, de una manera u otra, un estrato final que nos ha legado el proceso de transformación de la metafísica y ontología moderna. Este último nivel es facil de constatar en diferentes ámbitos de la producción filosofica de nuestro propio tiempo, aunque, sin duda, el lugar preferido es aquel que se refiere a la intersubjetividad trascendental lingüísticamente mediada, ya sea en el planteamiento apeliano o habermasiano, o cuando en los escritos gadamerianos se hace una referencia a la "Wirkungsgeschichtliches Bewusstsein», tanto como en aquellas otras y variadas ocasiones en las que se habla de conciencia lingüística e histórica.

Un proceso paralelo es el que han tomado las ciencias físico-matemáticas en su reduccion historicista, con lo que inevitablemente se pone la base teórica para un "retroceso" hacia las ciencias humanas y sociales, es decir, a las ciencias del espíritu, en las que el debate crucial no es otro que el de la conexión y dependencia entre conocimiento y valor, y con ello, la posibilidad de una fundamentación ética de la ciencia, al hilo de la validez o cientificidad de estas disciplinas .Pero, además, la necesaria vinculación que se da entre el problema de la fundamentación y la legitimación de los valores e instituciones, en una sociedad caracterizada en mayor o menor medida, pero siempre mirando hacia la posibilidad de racionalización, implica que el citado debate esté determinado no sólo por la filosofia y la ciencia, sino por toda una civilizacion occidental que ha llegado a convertirse y erigirse en civilación mundial. Sin embargo , si la cuestión relativa al fundamento último es, en primer lugar, una competencia filosófica, entonces parece que el destino de la civilización universal se decide en un ámbito aparentemente tan inocuo y aséptico, como es el de la filosofía. Pero no sólo es esto, ya que además, el filósofo en cuanto llega a cuestionarse el problema de los valores y de la validez, debe decidir, de alguna 
manera, el modelo de interpretación que, desde sí, debe dar sobre la cultura occidental. $Y$ es que, verdaderamente, la historia, en cuanto historia esencialmente occidental, remite al desarrollo de una racionalidad, cuya raíz pertenece a la filosofia griega, de manera que, además de una homogeneidad social entre la problemática filosofica y la institucional, tropezamos con otra homogenidad paralela, la que se da entre el sujeto de las ciencias del espíritu, y el sujeto de la historia que es, entonces, el objeto de una historiografía, muy próxima a lo que el propio Ortega llamaría "historiosofia».

Todas estas cuestiones y supuestos confluyen de forma natural en un elenco de preguntas acerca del sentido ontológico de la historicidad y sobre el tipo de experiencia filosófica que corresponde a la historicidad como un acto de autointerpretación del propio filósofo. Y, finalmente, cómo habrá de ser la forma de la institución de la misma filosofía, o si se prefiere, del pensamiento que ha de corresponder a este modo de experiencia. Evidentemente, las respuestas a preguntas como éstas sobrepasan, con mucho, la intención del presente trabajo. Por ello debemos limitarnos a esbozar mínimamente las líneas generales del problema que se desprenden de algunos de los textos y reflexiones heideggerianas que se erigen como materiales esenciales para el desarrollo del tema.

Pero dejemos ya todo este conjunto de consideraciones que hemos introducido en forma de premisas y requerimientos que nos orienten en el intrincado camino que hemos de recorrer para comenzar a reflexionar directamente acerca de la cuestion central que nos interesa. $Y$, así, preguntarse por el estatus ontológico del sujeto de la historicidad implica zambullirse directamente en el núcleo de la discusion filosófica contemporánea, de dos formas o sentidos distintos. Por una parte, tenemos aquél que remite a la cuestion del fundamento útimo en el que perviven y con el que conectan otros problemas paralelos como son los de la fundamentación de las ciencias humanas y sociales, tanto como el del carácter histórico de las ciencias fisico matematicas. En segundo lugar, está el sentido político y social ligado a la pérdida de referentes normativos, propia del relativismo, o, desde el lado contrario, al autoritarismo insidioso de la postura fundamentalista que no se resigna a desaparecer. Efectivamente, la discusión filosofica no se ha limitado a ubicarse dentro de las instituciones académicas, sino que, en ocasiones, en tanto afecta a la imagen que las sociedades tienen de sí mismas, llega a animar ciertos aspectos del debate político en los que se tropieza inevitablemente con cuestiones éticas y teóricas. La interconexión de la filosofía y la problemática social es, consecuentemente, más importante ahora que lo ha sido 
en otros períodos históricos, aun cuando parezca que ello tiene lugar en un momento en el que podríanse detectar ciertos atisbos de descomposición ética en el mundo occidental, y la debilidad con la que se configuran ciertas instituciones sociales, además de las insuficiencias de las que todavía adolece la propia Academia. Quizás sea por esta razon por la que se llega a negar la viabilidad de la tarea, propagándose rumores insistentes acerca de la inexistencia de valores objetivos, y que, en el mejor de los casos, las normas sólo tienen un cierto valor situacional con respecto al momento y al lugar en los que surgen.

Realmente, cuando se dice que toda norma o valor son meramente históricos, el concepto de "historicidad" funciona, en el fondo como una negación de validez, llegando a entender que el unico significado que corresponde al de validación es el de vigencia. Un principio es valido cuando tiene su vigencia y no es mera historia o pasado. Pero cuando esto sucede nos encontramos con que en el fondo se admite una exclusión recíproca total entre ambos conceptos, con lo que la historicidad conlleva en su interior la médula propia del «nihilismo». En definitiva, relativismo historico no es otra cosa que una figura concreta con la que se reviste el propio nihilismo, afectando a ciencias humanas y sociales, y donde, en último extremo, incluso podría desvanecerse la validez propia de las ciencias naturales, físico-matemáticas.

Por cuanto se refiere al sujeto de la misma historiciad, si existe un lugar que goce de algún privilegio que nos permita pasar, en lo referente a estas cuestiones, desde la pregunta a la respuesta, éste no puede ser otro que el de la actitud filosófica misma, frente a una sociedad industrializada que se mueve prioritariamente en unas coordenadas técnicas y estratégicas, con vistas a la instauración de una racionalidad instrumental. De hecho, una frase, como «la descomposición ética de occidentde», implica una interpretación y una valoración que se lleva a cabo desde los datos con los que se constituye la propia realidad social. Pero si, como sabemos, no existe ninguna interpretación que resulte totalmente objetiva, en el sentido de neutra, resulta que el destino de la actividad filosófica es el de llevarnos al fracaso por cuanto no puede en ningun caso gozar de la requerida independencia para proponer un veredicto eficaz y estable. Sin embargo, aun con todo, parece que sigue teniendo sentido la idea de que la filosofia como hecho historico como "res gestae», está obligada a decir algo con intencion y sentido radical.

Las ciencias humanas y sociales, en efecto, se han desarrollado al abrigo del descubrimiento de la "comprensión«(Verstehen), como método propio. Sin 
embargo, la comprensión tematiza, a su manera, la cuestión acerca de la subjetividad, tanto individual como colectiva, en cuanto agente de la historia, al cual remiten las creaciones socio-culturales que se producen en el devenir del tiempo. Tal como puede verse, hay una cierta homogeneidad, que ya había sido proclamada por Vico, entre el objeto y el sujeto de las ciencias en cuestión, lo que posibilita esa misma comprensión. Es el carácter subjetual de aquello que estudia el cientifico de las ciencias sociales, el cual por su parte tambien goza de su propio carácter subjetivo. Por otro lado, la radicalización del principio fundante, característico de la filosofía moderna occidental, lleva consigo un singular giro, el que va desde el sustancialismo u ontología realista a una ontología del sujeto epistémico que, poco a poco, se va determinando como una estructura en la que pueden advertirse diversos niveles o estratos de fundamentación, hasta alcanzar y llegar a una subjetividad histórica, próxima a la problematica de dichas ciencias de la comprensión, tal como ya se puede vislumbrar en el reconocimiento del apriori histórico y «mundo de la vida" tematizados por el último Husserl.

La constitución del sujeto de la historicidad supone, además de la "comprensión", la autocomprensión del científico social, como un agente histórico, de tal manera que se produce una fusión entre el sujeto y el objeto de las ciencias a las que nos referimos. Este sujeto, empero, es filósofo, en tanto que se interroga por la validez, que puede estar en contraposición con la simple "vigencia", y que conlleva una totalización del proceso histórico. En este caso, debe ser posible una segunda fusión que fundamente la totalización, a saber: aquella identificación de la filosofía y del filósofo como agentes históricos del proceso de racionalización occidental. Sólo entonces se puede entender que la autointerpretación del filósofo constituye el sujeto de la historicidad, pues racionalidad e historia llegan a estar aquí profunda y estrechamente ligadas.

El sujeto de la historicidad que se constituye y opera como depositario de la pregunta acerca de la validez radical, únicamente está facultado para fundamentar esta validez mediante una analítica del "sí mismo", como realidad existencial capaz de preguntar, en cuanto ente que formula una pregunta. Pero la respuesta a la pregunta incumbe e implica al ente interrogado, en un cierto respecto "es este ente». Sin embargo, el filósofo puede objetivarse a sí mismo de muy diversas maneras, como sujeto epistémico empírico como razón constituyente, o, incluso, como organismo autónomo con personalidad propia. El filosofo, si bien cuenta con la posibilidad de coincidir consigo mismo en cuan- 
to ente abierto a la validez, precisamente, por esta misma apertura, puede hacer efectivas otras posibilidades de no coincidencia.

Por tanto, parece imponerse con carácter prioritario, para el sujeto de la historicidad, detectar el "ser" de esta realidad existencial que es la historia y que posee su propia "entidad». De este modo, al preguntarnos y referirnos al estatuto ontológico del sujeto de la historicidad, planteamos el tema central que ahora nos ocupa. Sin embargo, si los significados básicos de la respuesta hay que ponerlos en el haber del ente que se pregunta por la validez, «en tanto que interroga y se vincula a la misma validez", por un lado, y al sentido de la temporalidad inherente a la historicidad, por otra, el hilo conductor del análisis únicamente puede consistir en una interpretación "temporal" de conceptos tales como el de "interpretación", "comprensión", "decisión", "proyección de sentidos", y otros similares. De esta guisa, nos vemos abocados directamente a la obra de M. Heidegger , en relación a la temática acerca del "tiempon, y que, como no puede ser de otra forma, no podemos ni siquiera desbrozar. Baste recordar esta cuestión recurriendo a las siguientes palabras escritas por J.Bengoa: "En "Sein und Zeit", el tiempo es comprendido unas veces como medio en el que se despliega o acontece toda investigación ontológica, y otras como principio intemporal de distinción de las distintas formas de ser. Según la primera concepción, toda comprensión se desarrolla necesariamente en el tiempo, es decir, está limitada a una época, y no puede superar sus límites. Según la segunda, una investigación ontológica que considera al tiempo como principio de distinción de los distintos modos de ser, se situaría ella misma más allá de los límites del tiempo. La primera sería la intención profunda de "Sein und Zeit". Pero la forma que esta obra adopta es manifiestamente la segunda: A todo lo largo de la misma no se hace otra cosa que intentar elaborar unas estructuras apriórico-formales del ser-ahí (los existenciales que son al ser ahí, lo que las categorías a los entes concretos) y cuyo carácter epocal, lejos de ser afirmado es excluido constantemente con la insistencia en su carácter "a priori”, y con el uso incesante del perfecto aprióricio (siempre ya)" ${ }^{1}$.

A tenor de lo recogido en el texto anterior, cabría decir que para su autor, se confunden dos cuestiones totalmente diferentes, aunque quizás fuera mejor decir que se "con-funden", es decir, que se mezcan y se compenetran. Sea cual

1 BENGOA, J., "De Heidegger a Habermas, Hermeneutica y fundamenticion última en la filosofia contemporánea ". Herder, Barcelona, 1.992, pp. 75-76. 
fuere el caso, conviene distinguir por una parte el carácter «temporal», es decir "datable" de la investigación desarrollada por M. Heidegger, y por otra, el rango ontológico de los fenómenos que su obra describe. Es cierto que «Sein und Zeitufue escrita en el año mil novecientos veintisiete, lo cual es un dato histórico constatable y comprobable, pero lo que no tiene sentido es decir que el propio "tiempo" es "temporal", o si se quiere que el "tiempo" tiene su propia "fecha». En consecuencia, parece que no debe haber lugar para ninguna objeción de principio, al hecho de que la estructura ontológica del "Dasain" con sus "existenciales» tenga un determinado estatuto. Otra cosa sería afirmar que el análisis concreto desarrollado en la obra pueda o no cuestionarse, modificarse, o perfeccionarse, en función de la legitimación dada por los fenómenos mismos.

Este tipo de extremos y cuestiones que acabamos de recordar, nos ofrecen la ocasión para plantearnos un problema verdaderamente esencial a la misma Ontología fundamental, como es el de establecer el supuesto, totalmente injustificado e injustificable de que el tiempo del "Dasein" es el tiempo del individuo, y por tanto un tiempo temporal, un tiempo inmerso en la temporalidad en general. Pero una vez que se acepta este postulado acerca de la individualidad del tiempo originario, el siguiente paso llevaría a negar la misma originariedad, ya que se trataría entonces de un tiempo meramente subjetivo, frente a la "objetividad" del tiempo universal. Y esta interpretación del tiempo está a la base del relativismo historicista, para quien la subjetividad hermenéutica es siempre una individualidad opuesta a la de otros individuos y en situación de irreductibilidad mutua. Si el sujeto de la historicidad representa el fundamento último, entonces no puede concebirse la posibilidad de una validez, sino únicamente meras opiniones, y en el mejor de los casos, la validez para un grupo histórico y social concreto, lo que no es otra cosa que lo que orteguianemente podríase denominar "vigencia». Como puede concluirse con facilidad, cuando no hay diferencia entre lo válido $\mathrm{y}$ no inválido, las escalas de valor pueden modificarse arbitrariamente y subjetivamente, con lo que, además, el sentido desfundamentador del concepto «historicidad «se hace proceder de esta construcción o forma de considerar ae tiempo. En consecuencia, la cuestión acerca del «ser del tiempo" concreta y puntualiza todavía más el núcleo del tema, ya que la pregunta sobre el estatuto ontológico del sujeto histórico solo se puede esclarecer si se la pasa por el cedazo de estas otras: ¿ Es el tiempo del sujeto de la historicidad un tiempo individual?, ¿tiene algún sentido hablar de una pluralidad de "Dasein«?, y, en definitiva ¿qué significa el "Mit-sein" como principio 
estructural y ontológico del "Dasein'? Ahora bien, antes de dar contestación a estas preguntas, se impone, explicar la raíz del supuesto tiempo universal en la temporalidad del hombre. Y, en efecto, si la esencia del hombre está conectada con la muerte, la estructura del tiempo que corresponde al hombre no puede ser otra que una línea infinita de puntos, el tiempo cosificado, es decir, espacializado, del sentido común. La llave para abordar el ser del tiempo y, por consiguiente, de la historicidad, apunta al concepto heideggeriano de "muerte", como fundamento de finitud. Por eso, precisamente, puede Heidegger dejar constancia de que el ser-para-la-muerte, es decir, la finitud de la temporalidad, se constituye como el fundamento oculto de la historicidad del ser-ahí ${ }^{2}$.

La muerte es el acabamiento del individuo y, consiguientemente, es el mejor argumento que confirma la propia subjetividad y pluralidad del tiempo. La finitud como esencia del tiempo legitima el relativismo de la interpretación individualista. La pregunta por el tiempo se transforma en pregunta por el "Mit-sein" y por el "Sein-zum-Tode", y la atención a temas tales como la historia, el tiempo, la individualidad del sujeto de la historicidad, la muerte, y otros, se convierten en criterio para valorar y medir la proximidad y lejanía de un autor a la problemática del propio sujeto de la historicidad.

Todo esto ilustra un hecho incontrovertible, y es que la filosofía ha de ir asimilada a un acto de autoconstitucion del filósofo, lo que equivale a una auténtica "conversión", a una experiencia del fundamento último desvelado en una actitud radical de preguntarse por la validez, por aquello con lo que nos vinculamos de forma absoluta. Sin embargo, si el fundamento último nos ha conducido al fenómeno de la muerte, a fin de ofrecernos en esta prueba suprema, la llave que esclarezca el sentido de la pregunta por la validez, ¿no tendríamos que experimentar el ser-para-la-muerte como un «acto» fundamental de autoconstitución de nuestra propia existencia histórica?, ¿̨no será la experiencia de la muerte, la primera y ineludible actividad del filósofo?

\section{Nudo}

Dos cuestiones pueden sorprender a quien se acerque a estas líneas. Por un lado el nombre que damos a esta segunda parte del trabajo, lo que, no es en

2 Vid. HeIdegGer, M., «Ser y tiempo», F.C.E., Mexico, 1.967, prg. 75 pp. 4l8-423. 
verdad especialmente relevante, ya que sólo hemos pretendido seguir una denominación clásica de presentación, nudo y desenlace que ahora traemos a nuestro propio discurso que, por otro lado, ha renunciado al etiquetado de una multitud de apartados y parágrafos. Mucho más interés puede tener el hecho de que ahora, sin previo aviso, procedamos a recordar y traer a mientes el discurso elaborado por W. Dilthey, acerca del ser de la historicidad, y del papel que el sujeto ejerce en las mismas ciencias del espíritu, cuando, hasta el momento, en líneas generales, lo que hemos hecho, ha sido establecer un primer contacto con el pensamiento de Heidegger, acerca de la temporalidad que le corresponde al Da-sein. Pero esto tampoco debe resultar extraño a quien esté familiarizado con el desarrollo textual de "Ser y tiempo", en donde tras la temática últimamente indicada, Heidegger establece un singular diálogo con el propio autor de la "Crítica de la razón histórica", por motivos que son expresados claramente en la primera de estas obras. En efecto, al comienzo del capítulo V, titulado "Temporalidad e historicidad», se destaca el hecho de que

«El análisis de la historicidad del Da-sein trata de mostrar que este ente no es temporal por estar dentro de la historia, sino que, a la inversa, sólo existe y puede existir históricamente por ser temporal en el fondo de su ser» ${ }^{3}$.

$Y$, a continuación, se indica que todas las consideraciones que siguen han de contentarse con señalar el lugar ontológico del problema de la historicidad, llevándose a cabo una especial apropiación y diálogo con la investigación diltheyana, que, a lo que parece, sigue teniendo su vigencia. Por eso, el autor, tras la exposición de cuestiones tales como la constitución fundamental de la historicidad, y la historicidad que corresponde al $\mathrm{Da}$-sein, pone de relieve la relación que tales desarrollos del problema tienen con las investigaciones historicistas de Dilthey ${ }^{4}$.

Apoyados, pues, en tales razones, además del hecho de que así lo exige el desarrollo mismo del tema, lo primero es recordar que la filosofía proyectada por W. Dilthey se puede caracterizar como un un especial enrejillado en el que confluyen todas las direcciones fundamentales del pensamiento moderno y contemporáneo, aun cuando, con ello,no tengan más remedio que que anu-

3 O.c. prg. 72 , p. 407.

4 Ibid. p. 408. 
larse y neutralizarse mutuamente. En primer lugar, hay que constatar que la exigencia de validez objetiva no puede exclusivizarse a la estricta esfera cognoscitiva, sino que debe hacerse extensiva a la totalidad del subjetividad humana, como también, y por ello mismo, deberá buscarse el fundamento no sólo a las ciencias explicativas de la naturaleza, sino también a las del espíritu. Por eso la filosofía debe propiciar un movimiento reflexivo de autoconocimiento que nos remita a un eje de fundamentacion universal y omnicomprensivo:

«Autognosis es conocimiento de las condiciones de la conciencia en las que se efectúa la elevación del espíritu a su autonomía mediante determinaciones de validez universal; es decir, mediante un conocimiento de validez universal, determinaciones axiológicas de validez universal y normas del obrar según fines de validez universal» 5 .

Ahora bien, aparte de otras notorias diferencias con el planteamiento trascendental kantiano, también considera W. Dilthey que esta fundamentación debe venir por vía inductiva, ya que el instrumento de la autognosis es la psicología que tiene por objeto propio el estudio de la mente de un sujeto empírico y concreto. Por eso, precisamente, se pronunciará repetidamente decantándose por la idea de que "la vida anímica singular, de índole individual y su desarrollo, es el material de investigación psicológica y su objetivo inmediato es la fijación de lo común en esta vida anímica de los individuos" ${ }^{6}$.

La radicalización de la vertiente empírica dará lugar a las corrientes positivistas y empiristas de las Ciencias humanas y sociales, y, consecuentemente, al relativismo de aquella opcion que pone el acento en la negación de toda validez. En cambio, la acentuación de los elementos de tipo trascendental conduce a través de posturas como la de $\mathrm{H}$. Rickert, a la experiencia teorética pura, como una forma de radicalización de la filosofía estricta, cuyo ejemplo más claro lo tenemos en E. Husserl. La dicotomía y contraposición binomial entre trascendentalismo y empirismo, forma un arco, en el que se ubican gran parte de los sistemas filosóficos del siglo diecinueve y principios del veinte, que se balancea entre esos polos, pero sin escapar nunca al dualismo que, de alguna manera, permanece incuestionado. El trascendentalismo salva la validez del fundamento, proyectándose hacia un universal abstracto y sacrificando la

5 DiLTHEY, W. "Sinopsis de mi filosofia", O.C. VIII,F.C.E. México, 1.978, pp.249-25O

6 DILTHEY, W., "Fundación de las ciencias del espiritu", O.C, vol. VII, p. 17. 
experiencia de la temporalidad, e ignorando el paso de la historia. Por su parte, el empirismo se zambulle irreflexivamente en la temporalidad aceptando de buen grado y conconfianza los hechos de la realidad concreta, pero sin pensar el tiempo como tal, y en el camino abandona la pretensión de validez universal entendiéndola como una abstracción ahistórica.

Conviene recordar que ya Hegel había captado el problema de la dicotomía universal- individual de manera mucho más profunda que sus sucesores filosóficos, pero otra cosa es que encontrase ya en su propio sistema una solución auténtica que disolviese la contraposición antinómica entre los dos extremos. Así es como puede hablar de que el espíritu de la historia de los pueblos constituye los momentos de un único espíritu universal, mediante el cual aquellos se elevan y se integran en una totalidad que se comprende a sí misma, algo que pone de relieve, entre otras, en su obra "Lecciones sobre la filosofia de la historia universals ${ }^{7}$. Lo universal se encarna en el particular, a través de la pasión, y

«si llamamos pasión al interés en el que la individualidad se entrega... y se fija en el objeto con todas las fuerzas de su voluntad, y concreta en este fin todos sus apetitos y energías, debemos decir que nada grande se ha realizado en este mundo sin pasión,...., el fin universal reside en los fines particulares y se cumple mediante ellos,..., el punto de vista de la finitud consiste en la actividad individual que da existencia a lo universal, realizando sus determinaciones» ${ }^{8}$.

Sin embargo, el universal no sólo aparece y se realiza gracias a la pasión, sino que también es el fruto de la moralidad y el deber social:

«El valor de los individuos descansa, pues, en que sean conformes al espíritu del pueblo, en que sean representantes de ese espíritu, pertenezcan a una clase, en los negocios del conjunto. Y para que haya libertad en el Estado es preciso que esto dependa del libre albedrío del individuo...La moralidad del indidividuo consiste,

7 HEGEL, G.W.F., "Lecciones sobre la filosofia de la historia universab. De. J.Gaos, Alianza, Madrid, 1.982, p. 46.

8 HEGEL, G.W.F., O.c., pp. 83-89. 
además, en cumplir los deberes de su clase. Y esto es cosa fácil de saber...Todo individuo tiene su clase y sabe lo que es una conducta justa y honrada" 9 .

Los mejores y más altos exponentes de la universalidad concreta son, por un lado, los héroes que «han sacado de sí mismos lo universal que han realizado", pero éste no ha sido inventado por ellos, sino que existe eternamente y se realiza a través suyo. Por otro lado, el Estado es la realidad en la que el individuo tiene y goza de su libertad, en cuanto sabe, cree y quiere lo universal. La unidad de lo concreto y de lo universal es la tensión que se encuentra entre ambos extremos en tanto que un principio superior, es decir, el de la validez se impone al inferior y le somete libremente. El individuo se universaliza realizando el acto ético que, objetivado como institución, o espíritu objetivo, crece y se desarrolla a lo largo de un proceso histórico. Pero Hegel quizás no llegue a explicar del todo y adecuadamente, según lo entendemos, ni el cómo ni el por qué ocurre esto, cuál es el punto de contacto ontológico entre la idea y el individuo: el sometimiento adopta, así, la forma de un milagro. En efecto, si el individuo es pura "empeiría", una "entidad» cerrada frente al universal, y si él mismo "narra" el universal, entonces ya no es individuo. El problema no se soluciona, sino que se traslada hacia otro lugar.

Esta cuestión que podríamos denominar "punto de contacto ontológico", conduce ya en la filosofía kantiana, al centro mismo de la problemática crítica, situándose en la deducción trascendental de las caregorías, y en la cuestión acerca de la raíz común de todas las representaciones conceptuales y sensibles, tal como se pone de relieve en el esquematismso trascendental. Sin embargo si se plantea la cuestión de una forma genérica, ¿no es verdad que este tema es una pregunta clave de la filosofía a lo largo de toda la historia, a partir de Platón, y su concepto acerca de la participación de las ideas?. Por cuanto se refiere a la filosofía contemporánea, recordemos por un momento los extremos «irresolubles» de la Teoría crítica habermasiana, que pretende una especial síntesis de lo trascendental y de lo empírico, lo que, tal como ya se ha dicho repetidas veces le conduce a una situación dilemática: "Al tratar de combinar un planteamiento trascendental de las condiciones objetivas del conocimiento con otro naturalista, Habermas parece verse atrapado en un dilema, o la natu- 
raleza tiene un status trascendental de una objetividad constituida, y por tanto no puede ser fundamento del sujeto constituyente, o la naturaleza es el fundamento de la subjetividad y no puede, por tanto ser simplemente una objetividad constituida" ${ }^{10}$.

La filosofía de la vida, por su parte, debería ser especialmente sensible al problema en el que se decide su propia legitimidad, y que conecta directamente con la cuestión que nos ocupa. Dice, por ejemplo Ortega, en un texto importantísimo de su trabajo "¿Qué es filosofian:

"Como nuestra realidad vivida es muy distinta de la realidad cósmica de la antigüedad, estará constituida por un conjunto de categorías o componentes, todos ellos forzosos igualmente originarios e inseparables entre sí. Estas categorías de "nuestra vida" es lo que buscamos. Nuestra vida "es la de cada cual", por tanto distinta la mía,de la tuya, pero ambas son "mi vivir» $\mathrm{y}$ en ambas habrá una serie de ingredientes comunes -las categorías de mi vida. Hay, sin embargo, para estos efectos una diferencia radical entre la realidad "mi vida" y la realidad "ser" que usa la filosofía. "Ser" de algo general que no pretende por sí mismo el carácter de lo individual. Las categorías aristotélicas son categorías del ser en general, pero "mi vida" aplíquese este nombre a mi caso o al de cada uno de Vds., es un concepto que desde luego implica lo individual; de donde resulta que hemos encontrado una idea rarisima que es a la par "general" e "individual". La Lógica hasta ahora ignoraba la posibilidad de un concepto en apariencia tan contradictorion ${ }^{11}$.

No podemos entrar a discutir todo el contenido de este fragmento que podría parecer harto singular y ambiguo, al menos para el que no esté versado en el pensamiento raciovitalista de Ortega. Con todo, parece que el autor propone un concepto de vida como una síntesis de universalidad e individualidad. Fundamenta esta pretensión diciendo que "mi", "tu", o "su" vida tienen unos ingredientes comunes, apelando a unas categorías que, a diferencia de las aristotélicas, no son remitidas a ninguna representación abstracta. Mas, sea cual fuere el caso, es que, al menos en intención, Ortega está persiguiendo ofrecer una explicación para superar la dicotomía metafísica entre las categorías de

10 MC. CARThY, "La teoria critica de J. Habermas", Tecnos, Madrid, 1.981, pp. 136-137.

11 ORTEGA Y GASSET, J., "¿Qué es filosofia?»,O.c. vol. II, Revista de Occidente, Madrid, 1.961, p. 427. 
individualidad y totalidad. Pero, volviendo ya al punto de partida de este "nudo", ¿se trata de algo que ya había presentido, o, por el contrario, solucionado,W. Dilthey, por su parte?

Dilthey retoma el problema de la subjetividad histórica respetando muchos de los requisitos a los que nos hemos referido más arriba. En primer lugar, considera y recaba para sí el carácter preteórico del sujeto, en contra de la consideración que de él se había llevado a cabo en la "Crítica de la razón pura». Si la subjetividad kantiana no es otra cosa que la estructura destinada a fundamentar la objetividad científica, y se determinaba por ser una síntesis apriori de intuiciones puras y categorías, es decir, de intuición y pensamiento, ahora hay que reconocer el mundo preteorético que posibilita la misma reflexión, dando una nueva dimensión a su horizonte de situación y actividad:

"Acerco cada elemento del actual pensamiento científico, abstracto, a la totalidad de la naturaleza humana, tal como la muestran la experiencia, el estudio del lenguaje y la historia, buscando la conexión entre ambos. $\mathrm{Y}$ así resulta que los principales elementos de nuestra imagen y nuestro conocimiento de la realidad, como la unidad de la vida personal, el mundo externo, los individuos fuera de nosotros, su vida en el tiempo y su interacción, pueden explicarse todos a partir de esta totalidad de la naturaleza humana en la que el querer, el sentir y el representar no constituyen más que aspectos distintos de su proceso real de vida. Las preguntas que todos hemos de dirigir a la filosofía no podrá responderlas el supuesto de un rígido apriori de nuestra facultad cognoscitiva, sino sólo la historia evolutiva que parte de la totalidad de nuestro ser" ${ }^{12}$.

Dilthey no se limita a remitirnos hacia la totalidad de la naturaleza humana, sino que señala las dimensiones preteoréticas de la conciencia como los auténticos fundamentos del entendimiento y de una razón que, en cierto aspecto, había sido divinizada por el pensamiento kantiano. Para él el a priori kantiano resulta rigido y muerto, en contra de los condicionantes reales de la conciencia que forman un proceso histórico vivo. Es preciso, pues, volver a la vida y a la historia, que es el auténtico caldo de cultivo desde el que, en realidad, pensamos ${ }^{13}$.Pero es posible entender estas condiciones bien, en términos

12 DiLTHEY, W, "Introducción a las ciencias del espiritu»,O.C. vol. I, p.6.

13 Vid. DiLTHEY, W., "Estructuración del mundo históricon, O.c. vol. VII, p. $13 \mathrm{O}$. 
ontológicos, en cuanto condiciones de posibilidad, bien como principios puramente empíricos, como condiciones ónticas. En cualquier caso, Dilthey apunta a la historia como fundamento que, según su método, ha de poder experimentarse en la vivencia del autoconocimiento. Efectivamente, el camino hacia el sujeto de la historicidad es la autointerpretación del propio "yo", que ahora se corresponde con la fundamentación de la filosofía como tal:

"Si abarcamos el problema del saber con esta amplitud, la solución del mismo en una teoría del saber puede designarse como autoconocimiento filosófico. Y este saber, en principio, es la tarea exclusiva de la parte fundamentadora de la filosofía” ${ }^{14}$.

En cierto sentido, puede decirse que Dilthey anticipa la exigencia de una inmediatez, un principio de todos los principios, idea que después desarrollará toda la Fenomenología, reclamando un positivismo radical que unicamente puede darse en la experiencia de sí mismo como sujeto. Por eso, llega a expresarse diciendo que la primera premisa de las ciencias histórica se encuentra en el horizonte de los hechos internos y en los estados psíquicos correspondientes. Esta es la única totalidad empírica de los hechos psíquicos y en ella se contiene todo el material para las demás experiencias mediatas ${ }^{15}$.Y para Dilthey esta inmediatez del sujeto "yo" se articula a la misma vivencia :

"Como tales, los contenidos de la conciencia, son tal como nos aparecen, y aparecen tal como son. Una percepción de un color, un dolor es, como hecho de nuestra conciencia inmediatamente dado... Un sentimiento es en la medida en que es sentido y es tal como es sentido: no son cosas diferentes la conciencia de él y su constitución, su «ser dado", y su "realidad»... La certeza de la vivencia no requiere ninguna mediación, y, por lo tanto, la vivencia puede ser calificada de inmediatamente cierta. Toda afirmación acerca de lo vivido es objetivamente verdadera si se halla en adecuación con la vivencia" ${ }^{16}$.

Sin embargo, si esta experiencia de la historicidad preteórica parece conducir directamente a la problemática del tiempo, por alguna razón que debería explicar-

14 O.C., vol. VII, p. 10.

15 Vid. O.C., vol. VII, pp. 16 y ss.

16 O.C., vol. VII, p. 26. 
se no se centra en un análisis determinativo de la experiencia del tiempo, entendida como condición necesaria de toda determinación de la historicidad. Si hemos de ser rigurosos y exigentes con el planteamaiento, hemos de considerar que, efectivamente, en Dilthey no hay una pregunta acerca de la temporalidad que justifique la importancia atribuida a la historia como fundamento del sujeto. En lugar de hacerse esta pregunta, que, bien planteada, podría haber dado juego a un desarrollo como el que depués llevará a cabo la Ontología fundamental, lo que se encuentra en su caso es la idea asegurada de la historia como una línea universal y una serie objetiva de presentes "substanciales", o, si se prefiere "fundamentales»:

«El curso empírico de la vida psíquica se compone de procesos, pues, cada uno de nuestros estados tuvo un comienzo en el tiempo, cambió durante él y se disipará con él” ${ }^{17}$.

No es, ni mucho menos, que Dilthey se haya olvidado o haya querido minusvalorar la importancia del tiempo, sino que, sorprendentemente, después de considerar que uen la vida tenemos, como su primera determinación categorial, la fundamentación de todas las demás» ${ }^{18}$, llega a expresarse sobre esta "determinación categorial" en unos términos que podríanse calificar de prefilosóficos, ya que están relacionados íntimamente con el desarrollo de la vida cotidiana, como cuando escribe que "así como una serie de cosas o de árboles se pierde y se empequeñece en la lejanía, así se escalona esta línea del recuerdo" ${ }^{19}$.

Tal como lo entiende este filósofo, los objetos se someten a categorías temporales como son la coetaneidad, la sucesión, la distancia temporal, la duración, o el cambio, principios que fundamentan la ciencia natural, pero, a la vez, todo este marco, en alguna medida kantiano, no agota la "vivencia» del tiempo, en la que se cumple, por fin, su propio concepto:

"En esta vivencia, el tiempo se experimenta como el avance incesante del presente, en el cual lo presente se está haciendo constantemente pasado, y lo futuro, presente" ${ }^{20}$.

17 O.C., vol. VII, p. 16.

18 DILTHEY, W. Estructuración del mundo histórico», O.C., vol. VII, p. 217 .

19 Ibid.

20 Ibid. 
Podría concluirse que, en cierto aspecto, Dilthey sigue siendo prisionero de una concepcion "espacial" de la propia temporalidad. Todo se entiende como una línea cuyo punto "cero" se encuentra entre un segmento negativo y otro positivo. En definitiva, se aproxima a un concepto de tiempo constuido desde la metafísica del espacio, de manera que el tiempo pasa a representarse como un movimiento, en el que el tiempo avanza, el móvil se desplaza de un punto a otro de la línea, y transforma el presente en pasado, y el futuro en presente. ¿Cómo plantear, entonces, la irreversibilidad y la finitud del tiempo que nos presiona y engulle? ¿No liquida la unidireccionalidad y finitud experienciales el valor explicativo y clarificador de la metáfora? ¿No confunde o falsea la imagen espacial, el sentido inmediato de la temporalidad, tal como ya considerara un pensador como $\mathrm{H}$. Bergson?

Dilthey, por su lado, deja abandonadas ciertas intuiciones fundamentales como son la exigencia de que el tiempo, como estructura originariamente subjetual, no puede identificarse con una entidad natural, un "medio" en el que la conciencia empírica se encuentra inmersa. Si así fuese, no habría un acceso inmediato al tiempo, sino a la vivencia subjetiva, individual del tiempo objetivo. Y, difícilmente, se podría hablar, entonces, del tiempo como una categoría determinante de la propia conciencia, sin naturalizarla y cosificarla. Sin embargo esto es, precisamente, lo que se hace, aun cuando ello tenga lugar sin las consecuencias que denunciaría E. Husserl en su "Filosofia como ciencia estrictos:

"Lo que caracteriza a todas las formas del naturalismo extremo y consecuente, que va desde el materialismo popular a las formas más recientes del monismo sensualista y del energetismo es, por un lado, la naturalización de la conciencia, y con ella de todos sus datos intencionles inmanentes, y, en consecuencia, de todo ideal y de toda norma absoluta» ${ }^{21}$.

Podríase, consiguientemente, admitir que Dilthey no es consecuente ni con el relativismo psicológico y naturalizador, ni con el trascendentalismo idealista , ni con ninguna línea que pretenda superar la dicotomía empirismo-trascendentalismo, lo que, en verdad, no quiere decir otra cosa que en su obra no

21 HuSSERL, E., "La filosofia como ciencia estricta». Trad. de E. Tabernig, Editorial Nova, Buenos Aires, 1.969, p. 50. 
se encuentren ninguno de estos extremos, sino todos ellos, a la vez, profundamente mezclados y confundidos en una especie de ambigüedad ontológica que no se logra superar. Por lo que hace a las anotaciones que podríanse calificar de preexistencialista, el propio Dilthey destaca lo siguiente:

"Cuando miramos el pasado nos comportamos pasivamente; se halla invariable; es inútil que el hombre aguijoneado por el recuerdo, se pierda en sueños acerca de lo que pudo haber sido de otra manera. Pero nuestro comportamiento, con respecto al futuro es activo y libre. Aquí, junto a la categoría de realidad, que emerge en el presente, surge la posibilidad. Nos sentimos en posesión de infinitas posibilidades" ${ }^{22}$.

De igual forma, es conveniente destacar que, a pesar de hablar de las infinitas posibilidades que tiene esta realidad, por otro lado, asocia el tiempo a la finitud y a la unidad del sujeto. La vida es lo que llena el tiempo,a la vez que toda su finitud se halla determinada por el mismo tiempo ${ }^{23}$. Sin embargo, sin insistir más en este tema, más importe parece recordar la relación que establece entre la temporalidad y la significatividad, una idea que será desarrollada ampliamente por el planteamiento ontológico existencial:

"La categoría de significado designa la relación de las partes de la vida con el todo, que está fundado en la naturaleza de la vida. Poseemos esta conexión tan sólo por medio del recuerdo, en el que podemos contemplar el curso transcurrido de la vida...Todo plan de vida es expresión de una captación del significado de la vida. Lo que nosotros establecemos como fin para el futuro, condiciona la determinación del significado del pasado» 24 .

A pesar de que Dilthey acabe remitiendo la totalización del pasado al recuerdo, y por tanto, a la conciencia como memoria, y no al "para" de la red significativa proyectiva, puede rastrearse y adivinarse en él el convencimiento de una vinculación entre el proyecto o "determinación del fin" y la significación del pasado, que anticipa una posible totalización de carácter preconsciente y preteorética. Y, finalmente, antes de concluir este «nudo", no está de más recordar el reconocimiento diltheyano del fracaso académico acerca de su reflexión sobre el

22 DILTHEY, W., "Estructuración del mundo histórico». O.c. vol. VII, p. 218.

23 DILTHEY, W., Vid. O.C., vol. VII, p. 254.

24 DiltheY, W., O.C. vol. VII, p. 258. 
tiempo, lo que acaba abonando el terreno para que se releguen estos aspectos a las corrientes irracionalistas que seguirán presentes en el siglo veinte. Para Dilthey, las antinomias que el pensamiento encuentra en la vivencia del tiempo surgen de su impenetrabilidad para el conocimiento.

\section{Desenlace}

A partir de Dilthey, la opción que, en principio, podía resultar más acerta$\mathrm{da}$, desde un plano teórico, para dar respuesta al problema acerca del papel de la reflexión y del papel del filófoso como funcionario de la humanidad, y, consiguientemente también al destino de Europa, es la adoptada por E. Husserl, pero que a su manera es anticipada por Heinrich Rickert en su sentido «normativizador" $\mathrm{y}$ «estabilizador»:

"Hubo que tornar consciente aquí de modo expreso sólo la esencia sistemática y al mismo tiempo críticamente valorativa del tratamaiento filosófico del universo histórico, y esto sólo puede quedar oscuro alli donde se confunden ser real y ser irreal, realidad y valor, o donde, debido a la desconfianza reinante para con una filosofía científica de los valores, sólo se ose aplicar veladamente los supuestos axiológicos previos que se utilicen a fin de dar la apariencia de un tratamiento puramente contemplativo, es decir, libre de valores» 25 .

Rickert es consciente de que en esta decisión se juega la autointerpretación del hombre y el destino de la humanidad, especialmente el de la sociedad occidental, y por eso añade:

«La búsqueda de los supuestos axiológicos previos y la comprobación de su necesidad absoluta como principios, se torna una imperiosa obligación para la filosofía, especialmente debido a la oscuridad y a la nebulosidad que...reina en ese campo. Quizás se rechace también el reconocimiento de valores formales como universalmente válidos pero entonces deberíamos dejar claramente establecido que con ello nos quedamos atascados en el historicismo, es decir, en el nihilismo" ${ }^{26}$.

25 RICKERT, H. "Introducción a los problemas de la filosofia de la historia" Editorial Nova, Buenos Aires, 1.961, p. 137.

26 Ibid. 
Sin embargo, el camino más prometedor hacia la verdad sería el paso del «ser real al ser ideal", mediante la reducción "eidetica» y, consecuentemente, gracias a una operación con un sentido existencial muy claro, -la irrealización-. Pero tal "operación" expresará el fracaso de todo academicismo y cientifismo ante la "experiencia del tiempo", y el abandono de este campo a las sucesivas oleadas con que embiste el irracionalismo. Las palabras de Husserl, al respecto, son harto significativos. Recordemos como muy apropiado el texto siguiente:

«El yo , el ego trascendental es lo que precede a todo lo mundano. Así pues, el yo constituyente no es idéntico al yo-mundo, a mí mismo en tanto que realidad psicofísica. Mi vida de conciencia anímica, psicofísica y mundana no es idéntica a mi Ego trascendental en el que se constituye para mí el mundo con todo lo físico y lo psíquico" ${ }^{27}$.

La cuestión reside, entonces, en preguntarse si hay entre el irracionalismo y el racionalismo del ser ideal, un camino eficaz que permita el alumbramiento de una ontología de la existencia histórica realmente eficaz, en la que la vida y la verdad se encuentren hermanadas y firmen un pacto definitivo. Este camino es el que, propiamente, inicia M. Heidegger con la publicación de «Ser y tiempo" en 1927, aun cuando en cierto aspecto el "Da-sein" siga siendo interpretado a la manera de una especial individualidad, lo que podría llegar a borrar y hacer desaparecer el sentido filosófico general que se pretende en la obra. Hay efectivamente fragmentos que quizás confirmarían esta especie de "recaída" o falta de separación radical con aquellos enfoques que pretendían ser superados:

"Pero si el "Ser ahr" que es en forma de "destino individual", existe en cuanto "ser en el mundo", esencialmente en el "ser con otros", es su gestarse histórico un gestarse con y constituido como destino colectivo. Con esta expresión designamos el gestarse histórico de la comunidad, del pueblo. Tal "destino colectivo" no es un conjunto de "destinos individuales", como

27 HUSSERL, E., «Logica formal y trascendental", prg. 96-a, Hua XVII, p. 245. Edicion castellana en U.A.Mexico, 1.962, p. 249.- En el prefacio para la edición inglesa de «Ideen», escribiría: La subjetividad trascendental es un dominio absolutamente independiente de experiencia directa...La subjetividad pierde precisamente lo que hace de ella algo real en el mundo que se extiende delante de nosotros...Yo no soy ya entonces un sujeto humano en el mundo universal, existencialmente puesto". 
tampoco puede concebirse el "ser uno con otro" como un venir a estar juntos varios sujetos. En el "ser uno con otro" en el mismo mundo y en el "estado de resuelto" para determinadas posibilidades son ya trazados por anticipado los "destinos individuales" "28.

Sea cual fuere el caso y la situación final del problema en el desarrollo heideggeriano, hay que considerar la posibilidad de una síntesis especial entre lo individual y lo trascendental, entre lo existencial y esencial, formulandose por la conveniencia de una singularidad trascendental, que aún cuando lo llevara a cabo de forma paradojica, reuna en sí ambos aspectos. Pero esta posibilidad sólo puede tener lugar mediante una redefinición del sentido del tiempo, por cuanto se refiere a la dicotomía universal-particular. $Y$, para ello, es posible que el mejor camino sea el de intentar una nueva lectura de la Estética trascendental kantiana, lo que nos ayudaría a entender que el fenómeno del tiempo no es un objeto individual y que, además, la misma palabra del tiempo no se refiere a ningún género abstracto, a ninguna clase universal. El tiempo es, a la vez, individual y universal,al ocupar un puesto central y sintetizador de toda la estructura apriórica de la subjetividad trascendental.

Pero, de este modo, la tarea que se nos presenta si bien importantísima,es imposible iniciar, y llevar a buen fin, ahora. Muchos textos y aspectos deberían tenerse en cuenta para traer a mientes el significado y el papel sintético a priori de la temporalidad en el pensamiento kantiano, lo que conduciría inevitablemente a tenerse que reconocer que con ello se está proponiendo, aun cuando no se tenga que aceptar integramente la definicion y todas las impli-

28 HeidegGer, M., Ser y tiempo. Ed.cit. prg. 74, p. 415.-En "El concepto de tiempos se había expresado de la siguiente manera: “El ser-ahí, en tanto que este ser-en-el-mundo, es justamente un ser-con, un ser con otros; lo cual significa tener ahi con otros el mismo mundo, encontrarse recíprocamente, ser con otros en el modo del ser-uno-para-otro. Pero, a la vez, este ser -ahí está presente ante los otros como si fuera una cosa, a la manera de una piedra que está ahí sin tener un mundo ni cuidarse de él" Ed. Trotta, Madrid,1.995, pp. 35-36.. Afirmaciones éstas que se encuentran bastante alejadas de las que, situándose en una instancia distinta, corresponden a su etapa última, tales como: "El ser no es ninguna cosa real y concreta, y por tanto nada temporal, mas es, empero, determinado como presencia por el tiempo. El tiempo no es ninguna cosa real y concreta, y por tanto nada ente, pero permanece constante en su pasar, sin ser él mismo algo temporal como lo ente en el tiempo. Ser y tiempo se determinan recíprocamente, pero de una manera tal que ni aquél, el ser, se deja apelar como algo temporal, ni éste, el tiempo, se deja apelar como ente. Al cavilar sobre todo esto, nos sorprendemos vagando errráticamente entre enunciados contradictorios". "Tiempo y ser", Tecnos, Madrid, 1.999, p.22. 
caciones que el tema tiene para Kant, una via de superación de la dicotomía existente entre la individualidad y la universaliddad, entre la vida concreta y el discurrir teórico, sin tener que rechazar ninguno de los dos extremos. Cuando nos referimos al tiempo, tal como lo piensa Kant, hay que reconocer en él dos dimensiones insoslayables aunque parezcan contradictorias: su realidad empírica y su idealidad trascendental ${ }^{29}$. Por esta linea de investigación Kant anuncia, especialmente en la primera edición de su "Crítica» una interesante línea de trabajo, que merecería la pena ser continuada para sacar de ella todas sus virtualidadades, pero que ahora no podemos abordar por la complejidad de sus dimensiones, que exceden con mucho a nuestros propósitos, a la hora de redactar estas reflexiones. 